\title{
Cognitive impairment in patients with severe psoriasis
}

\author{
Luiza Marek-Józefowicz ${ }^{1}$, Marcin Jaracz ${ }^{2}$, Waldemar Placek³ ${ }^{3}$ Rafał Czajkowski ${ }^{1}$, Alina Borkowska ${ }^{2}$
}

${ }^{1}$ Chair of Dermatology, Sexually Transmitted Diseases and Immunodermatology, Faculty of Medicine in Bydgoszcz, Nicolaus Copernicus University in Torun, Poland

${ }^{2}$ Chair and Department of Clinical Neuropsychology, Faculty of Health Science in Bydgoszcz, Nicolaus Copernicus University in Torun, Poland

${ }^{3}$ Department of Dermatology, Sexually Transmitted Diseases and Clinical Immunology, Faculty of Medical Sciences,

University of Warmia and Mazury, Olsztyn, Poland

Adv Dermatol Allergol 2017; XXXIV (2): 120-125

DOI: https://doi.org/10.5114/ada.2017.67074

\begin{abstract}
Introduction: Psoriasis is a chronic inflammatory skin disease, in which an important role is played by psychological factors.

Aim: To evaluate the frontal cognitive functions in patients with psoriasis.

Material and methods: The study included 188 subjects (97 patients with psoriasis and 91 healthy controls). To assess the dorsolateral prefrontal cortex functions, the Trail Making Test and the Stroop test were applied. Severity of psoriasis was assessed by means of the PASI index.

Results: Compared to healthy subjects, psoriatics scored lower in neuropsychological tests assessing memory and executive functions.

Conclusions: Cognitive dysfunction disclosed by neuropsychological assessment of frontal functions was evident in patients with psoriasis.
\end{abstract}

Key words: psoriasis, cognitive functions, executive functions, depression, prefrontal cortex.

\section{Introduction}

Psoriasis is a chronic disease significantly impairing psychosocial functioning of patients, and is considered to be a severe psychosomatic disease. The results of current research indicate that psychological factors play an important role in the etiology of many somatic diseases. The need to take psychological factors into account for dermatological disorders has been increasingly emphasized [1-6].

Cognitive functions enable one to adapt to shifting environmental conditions. They are important in perception, judgment, and decision-making, as well as building self-esteem. They include information processing, attention, learning, language abilities, memory and complex mental processes, such as executive functions. In coping with the disease, particularly relevant are decisionmaking processes related to the activity of several brain structures, especially those determining information processing, evaluation and understanding of the different situations, and emotional control. Based on the data from neuroimaging studies, it has been concluded that the main structures of the brain, important for decisionmaking, are ventromedial and dorsolateral prefrontal cortex. The ventromedial prefrontal cortex is responsible for the emotional aspect of decision-making, and many studies show that a final decision is associated with the activity of this structure. The dorsolateral prefrontal cortex is responsible for the analysis of problematic situations, understanding and validation of information pouring in from our surroundings and their quick integration [7]. In these processes, a key role is played by executive functions, which are based on working memory. They are essential for the proper conduct and integration of complex cognitive processes such as planning, conceptual thinking, problem solving, understanding of the situation, and adaptation to changing environmental conditions. Functioning of the working memory is mainly dependent on the activity within the dorsolateral prefrontal cortex. Due to its connection with the limbic system and the subcortical structures (as well as other areas of the cerebral cortex), this area is responsible for the most complex cognitive and emotional functions.

Address for correspondence: Luiza Marek-Józefowicz MD, Chair of Dermatology, Sexually Transmitted Diseases and Immunodermatology, Faculty of Medicine, Nicolaus Copernicus University, 9 Sklodowskiej-Curie St, 85-094 Bydgoszcz, Poland, e-mail: lui06@interia.pl

Received: 14.02.2016, accepted: 3.04.2016. 
Working memory is a short-term memory, which allows for the correct integration of information, depending on the requirements of a given situation, which is possible due to the "online processes". It is also responsible for timed storage of information about current activities and the ability to switch to a new way of acting [8]. Evaluation of the efficiency of working memory is being carried out with specific neuropsychological tests, designed for the assessment of its various aspects.

In the research on psoriasis, attention tends to be drawn to the greater prevalence of depressive symptoms, which may impact the efficacy of cognitive functions [911]. Many studies indicate that symptoms of depression are associated with a decrease in psychomotor speed, impaired attention as well as with disturbances of memory and executive functions, related to hypofrontality in course of the depressive state [12-14].

\section{Aim}

The aim of this study was to evaluate prefrontal cognitive dysfunctions in patients with severe psoriasis (PASI $>10$ points) in the context of demographic and clinical factors, as well as depressive symptoms.

\section{Material and methods}

\section{Subjects}

Ninety-seven patients with psoriasis (62 men and 35 women) hospitalized in the Department of Dermatology in Bydgoszcz, Poland, were enrolled in the current study. The patients' age ranged from 18 to 60 years (mean: $44.1 \pm 13.0$ years). The control group consisted of 91 healthy individuals (39 men and 52 women), aged 22-65 years (mean: $41.0 \pm 10.7$ ). Exclusion criteria were: age over 65 and under 18 years, history of head injuries, comorbid severe neurological and autoimmune diseases, drug or alcohol addiction, intake of immunosuppressive drugs in the past 3 months, and treatment with oral agents for psoriasis in the past three months. Examination and assessment of severity of dermatological lesions (Psoriasis Area and Severity Index - PASI) was conducted by one physician, which was also the case during the administration of TMT and Stroop tests. The diagnosis of psoriasis was based on the clinical picture and data from an interview. When necessary, a biopsy was performed for histopathological evaluation.

The study was approved by the Nicolaus Copernicus University Torun, Collegium Medicum Bydgoszcz Research Bioethics Committee. All subjects gave their written consent to participate in the study after the nature of procedures was fully explained to them.

\section{Methods}

\section{Psoriasis Area and Severity Index (PASI)}

This scale, developed by Fredrikson and Pettersson in 1978, is one of the most commonly used tools for the evaluation of psoriasis severity. The value is the sum of the products of PASI for the four parts of the body. PASI maximum value is 72 , the minimum 0 . Higher scores indicate greater severity of the clinical lesions $[15,16]$.

\section{Trail Making Test (TMT)}

The test consists of two parts, A and B. In part A the subject is asked to connect, as quickly as possible, a series of circles numbered 1 to 25 , in a numerical order. In part B, the task of the subject is to connect alternately numbered circles marked with letters as quickly as possible, according to the formula: 1-A 2-B-3-C, etc. Part A examines psychomotor speed, and the efficiency of visual spatial coordination, part B is a measure of visual-spatial working memory and set shifting [17]. The time of performance in both parts of the test was analyzed in the current study.

\section{Stroop Color-Word Interference Test}

This test is designed to evaluate verbal working memory and attention efficiency. It consists of two parts: RCNb (Reading Color Name In Black) and NCWd (Naming Color of Word-Different). The first part of the test requires the subject to read, as quickly as possible, words denoting colors which were printed black on white. In the NCWd part, the subject is asked to call as quickly as possible the color of the words' print. Print color does not coincide, however, with the color, which name is written. Here, after learning an initial criterion for action, the subject needs to switch to another, while the previous criterion is still remembered [18]. The time of performance in both parts of the test was analyzed in the current study.

\section{Beck Depression Inventory (BDI)}

The scale consists of 21 items relating to various symptoms of depression. Within each category, there are four possible answers indicating the intensity of the symptoms, starting with its absence (0) up to very high severity (3). The rating assigned to each response ranges from 0 to 3 points. Minimum overall score is 0 points, and maximum is 63 points. Threshold for the recognition of depression was adopted as 12 points [19, 20].

\section{Statistical analysis}

The distribution of variables was assessed by means of the Shapiro Wilk test. As the distribution of variables tested did not meet the normality criterion, nonparametric tests were applied for the statistical analysis. The significance of differences between groups was assessed by means of the Mann Whitney U-tests and Spearman's 
Table 1. The results of TMT-A and B, Stroop A and B tests in patients with psoriasis and healthy controls group

\begin{tabular}{lcc}
\hline Test & $\begin{array}{c}\text { Patients with psoriasis } \\
\text { Mean value; } 25-75 \%\end{array}$ & $\begin{array}{c}\text { Healthy controls } \\
\text { Mean value, 25-75\% }\end{array}$ \\
\hline TMT A & $36.0 ; 29.0-44.0$ & $21.0 ; 17.0-28.5^{\star *}$ \\
\hline TMT B & $65.0 ; 55.0-78.0$ & $40.0 ; 33.0-53.0^{\star *}$ \\
\hline Stroop A & $36.0 ; 31.0-45.0$ & $21.0 ; 17.0-28.0^{\star *}$ \\
\hline Stroop B & $60.0 ; 55.0-69.0$ & $47.0 ; 38.0-55.0^{\star *}$ \\
\hline Beck Depression Inventory & $13.0 ; 9.0-18.0$ & $2.0 ; 0.0-5.0^{\star *}$ \\
\hline
\end{tabular}

Significant differences between the groups ${ }^{* *} p<0.001$, U-test of Mann-Whitney.

Table 2. Correlations between the results of neuropsychological tests and the severity of depressive symptoms on Beck Depression Inventory, and the age and level of education, in the group of patients and healthy subjects (Spearman's rho)

\begin{tabular}{|c|c|c|c|c|}
\hline \multirow[t]{2}{*}{ Test } & \multicolumn{2}{|c|}{ Age } & \multicolumn{2}{|c|}{ Level of education } \\
\hline & Patients with psoriasis & Healthy controls & Patients with psoriasis & Healthy controls \\
\hline TMT A & $0.38^{*}$ & -0.03 & $-0.28^{*}$ & $-0.29^{*}$ \\
\hline TMT B & $0.27^{\star}$ & $0.40^{*}$ & -0.16 & $-0.29^{*}$ \\
\hline Stroop A & $0.43^{*}$ & -0.04 & $-0.30^{*}$ & $-0.43^{*}$ \\
\hline Stroop B & $0.32^{*}$ & 0.11 & -0.17 & $-0.41^{*}$ \\
\hline $\begin{array}{l}\text { Beck Depression } \\
\text { Inventory }\end{array}$ & -0.02 & 0.16 & -0.02 & -0.15 \\
\hline
\end{tabular}

*Significant correlation at $p<0.05$.

rho coefficient was applied for the assessment of correlations between variables.

\section{Results}

The PASI score in patients enrolled fell in the range of 10.1-58.7 points, with mean $21.9 \pm 9.4$ SD points.

The results of the neuropsychological tests in patients with psoriasis were significantly worse than those of the healthy controls. Afflicted subjects presented significantly lower psychomotor speed (TMT test A), diminished efficiency of spatial working memory and set shifting ability (TMT B), as well as poorer verbal working memory and attention, as evidenced by the longer time of performance in Stroop Test A and B (Table 1).

There was a significant correlation between the results of neuropsychological tests, and the age of the respondents in both investigated groups. Lower results obtained in neuropsychological tests were associated with higher age both in psoriasis patients and healthy control groups. A higher level of education correlated with better performance in neuropsychological tests, except for no association between the level of education and the Stroop B test results in the psoriasis patients group (Table 2 ).

In the patient group there was no association between the severity of psoriasis changes indicated by the

Table 3. Correlations between neuropsychological test results and severity of psoriasis in PASI score and duration of the disease and last exacerbation in the group of patients with psoriasis (Spearman's rho)

\begin{tabular}{lccc}
\hline Test & PASI & Duration of illness & Duration of last exacerbation \\
\hline TMT A & 0.08 & $0.22^{*}$ & -0.16 \\
\hline TMT B & 0.05 & $0.22^{*}$ & -0.13 \\
\hline Stroop A & 0.18 & $0.35^{*}$ & -0.17 \\
\hline Stroop B & 0.08 & 0.16 & -0.16 \\
\hline
\end{tabular}

*Significant correlation at $p<0.05$. 
PASI score, duration of the last exacerbation of the disease and the results of neuropsychological tests. Longer duration of the disease correlated with worse neuropsychological performance, except for the results of Stroop B Test (Table 3).

\section{Discussion}

The main finding of the present study is that patients with psoriasis present impairment of working memory, when compared to healthy controls. They perform significantly worse in neuropsychological tests measuring different aspects of working memory, than the healthy control group, which indicates a dysfunction of the prefrontal cortex in these patients. This dysfunction may be important in the etiology of many psychosomatic diseases, including psoriasis and affective disorders and may be associated with abnormal activity of neurotransmitter systems in the brain. Disorders associated with cognitive impairment, such as schizophrenia and affective disorders, have been found to be associated with dysfunction in various neurotransmitter systems, crucial for the efficient activity of the prefrontal cortex of the brain, including dopaminergic, serotonergic, GABAergic and noradrenergic systems. These systems play an important role in cognitive and emotional processes as well as the resistance to stress $[21,22]$. Perhaps cognitive dysfunctions in psoriasis have similar determinants.

Results of the present study also indicate a significant prevalence of depression in psoriasis patients, which in over half of the studied sample reached the clinical level. In these patients, BDI scores were between 12 and 32 points, with means intensity of depressive symptoms of 18.2 points. The BDI results correlated positively with psoriasis severity and negatively with duration of illness, which may be the effect of adjustment to the disease and coping with its psychosocial consequences. This confirms that psoriasis and depression may cooccur. Moreover, the severity of depressive symptoms in some patients indicate the need for their better recognition and plausibility of anti-depressive treatment. These results are concordant with those obtained by the German authors who observed a larger prevalence of depression, as well as the relationship between its severity and quality of life in patients with psoriasis [23].

Compared to healthy controls, psoriasis patients show significantly worse results (longer time of performance) in both parts of the TMT test used for the evaluation of visuo-spatial working memory. While the level of TMT A performance in psoriasis patients indicates psychomotor slowness, worse results in the TMT B are connected with prefrontal cortex disturbances. These result in poorer set shifting and cognitive control, poorer visuospatial memory, and impairment of executive control. Similarly, in terms of verbal working memory, they ob- tained worse results in the Stroop Test (especially part B), compared to healthy subjects.

Contrary to our hypotheses, the results obtained by psoriasis patients in all neuropsychological tests were not related to the severity of depressive symptoms, such relationship being observed in patients with the diagnosis of depression in the course of affective disorders [12]. No relationship was also found between the efficiency of cognitive functions and severity of psoriasis indicated by the PASI score. Likewise, no such relationship was found with the duration of the last exacerbation of the disease.

Interestingly, longer duration of psoriasis correlated with significantly worse results in neuropsychological tests. This may indicate progressive changes within the central nervous system (CNS), especially in the prefrontal cortex. This phenomenon is difficult to interpret, however the adverse effects of inflammatory factors (cytokines, cortisol) on the nervous system during the course of the disease have one possible cause. It was proven, in the affective disorders, that cortisol can have neurotoxic effects, especially for the hippocampal neurons, involved in the declarative memory and emotional processes.

This hypothesis is supported by data from multiple studies that established that the nervous, immune and endocrine systems behave as a functional unity. These systems communicate and interact with each other using neurotransmitters, hormones, or cytokines, problems in the one of them affecting the functioning of the others $[24,25]$. Damage to the nervous system, especially to the hippocampus and the hypothalamus, affects the function of the thymus and production of T cells. Moreover, cytokine receptors are present together with neurotransmitter receptors on the same cells. Receptors for interleukin (IL)-1, IL-2, and IL- 6 are present within the structure of the hypothalamus and the hippocampus, IL-1, and IL- 6 are continuously produced at low concentrations in neurons and glia. In the course of somatic disorders, in which increased synthesis of pro-inflammatory cytokines is observed, symptoms of depression or a higher risk of developing symptoms of depression, were documented. The depression cytokines modulate the function of the brain, affecting sleep patterns, appetite, and cognitive functions $[26,27]$. The hypothalamic-pituitary-adrenal (HPA) axis is stimulated on all levels by proinflammatory cytokines. At the same time glucocorticoids secreted due to the stimulation of the limbic-hypothalamic-pituitaryadrenal (LHPA) axis interact with immunosuppressants [28-30]. Both in psoriasis and depression, pathological proinflammatory immune readiness has been observed $[31,32]$. Finally, on the psychological level, early onset of the skin disease can be an element of dysfunctional depressive cognitive schema, and thus may facilitate the occurrence of depression.

One of the factors of protecting properties to cognitive functions, is cognitive activity. The current study demonstrated a relationship between higher levels of 
education and better efficiency of the tested functions, especially in healthy subjects. In the patient group, a significant correlation between education and neuropsychological test results was observed, however, it pertained only to the two tests, i.e. Stroop Test Part A and Part A of the TMT test. While psychomotor speed and overall verbal functions efficiency in psoriasis patients are associated with a higher level of education, this relationship was not observed in the case of complex executive frontal functions measured by Stroop B and TMT B tests. The performance in these tests requires efficient working memory and executive functions associated with the prefrontal cortex activity. While higher levels of education correlated with higher efficacy of these functions in healthy subjects, this effect may be hampered by the psoriasis-related dysfunctions of prefrontal cortex in our group of patients.

Moreover, according to the current data, etiology of depression and cognitive dysfunctions is associated with abnormalities in similar brain structures, such as prefrontal cortex and hippocampus. It may also explain the high incidence of neuropsychiatric symptoms in psoriasis, namely the anxiety, irritability and depressive symptoms associated with CNS dysfunction [33]. This suggests potential usefulness of mental training, e.g. neuropsychological therapy, in patients with psoriasis whose cognitive functions are impaired.

Stress and depression accompanying psoriasis are well-recognized risk factors for cardiovascular events and thus may increase the risk of developing dementia. This is especially important for older patients, in which the effect of aging on cognition can be accompanied by the aforementioned risk factors. This indicates the plausibility of early screening for the symptoms of mild cognitive impairment $(\mathrm{MCl})$, which are considered an intermediate stage between normal aging and dementia, especially in older patients (60-65 years old), experiencing subjective decline in intellectual fitness [32]. This can be done using standard screening tools, such as the Mini-Mental State Examination (MMSE). Inconclusive results can be accompanied by full neuropsychological examination.

\section{Conclusions}

Patients with psoriasis show a significant impairment of prefrontal neuropsychological functions, which are independent from the level of depression and severity of the illness.

\section{Conflict of interest}

The authors declare no conflict of interest.

\section{References}

1. Mackenna RMB. Psychosomatic factors in cutaneous disease. Lancet 1994; 267: 679-81.
2. Gupta MA, Gupta AK. Psychodermatology. An update. J Am Acad Dermatol 1996; 34: 1030-46.

3. Shenefelt PD. Psychodermatological disorders: recognition and treatment. Int J Dermatol 2011; 50: 1309-22.

4. Wilson PB, Bohjanen KA, Ingraham SJ, et al. Psoriasis and physician activity: a review. J Eur Acad Dermatol Venereol 2012; 26: 1345-53.

5. Ograczyk A, Miniszewska J, Kępska A, Zalewska-Janowska A. Itch, disease coping strategies and quality of life in psoriasis patients. Postep Derm Alergol 2014; 31: 299-304.

6. Tsai TF, Wang TS, Hung ST, et al. Epidemiology and comorbidities of psoriasis patients in a national database in Taiwan. J Dermatol Sci 2011; 63: 40-6.

7. Rolls ET, Grabenhorst F. The orbitofrontal cortex and beyond: from affect to decision-making. Prog Neurobiol 2008; 86: 216-44.

8. Baddeley A, Della Sala S. Working memory and executive control. Philos Trans R Soc B 1996; 351: 1397-403.

9. Richards HL, Fortune DG, Chong SL, et al. Divergent beliefs about psoriasis are associated with increased psychological distress. J Invest Dermatol 2004; 123: 49-56.

10. Kotrulja L, Tadinac M, Joki-Begi NA, Gregurek R. A multivariate analysis of clinical severity, psychological distress and psychopathological traits in psoriatic patients. Acta Derm Venereol 2010; 90: 251-6.

11. Rieder E, Tausk F. Psoriasis, a model of dermatologic psychosomatic disease: psychiatric implications and treatments. Int J Dermatol 2012; 51: 12-26.

12. Borkowska A, Rybakowski J. Neuropsychological frontal lobe tests indicate that bipolar depressed patients are more impaired than unipolar. Bipolar Disord 2001; 3: 88-94.

13. Martínez-Arán A, Vieta E, Reinares M, et al. Cognitive function across manic or hypomanic, depressed and euthymic states in bipolar disorder. Am J Psych 2004; 161: 262-70.

14. Arts B, Jabben N, Krabbendam L, Van Os J. Meta-analyses of cognitive functioning in euthymic bipolar patients and their first-degree relatives. Psychol Med 2008; 38: 771-85.

15. Fredriksson T, Petersson U. Severe psoriasis-oral therapy with a new retinoid. Dermatologica 1978; 157: 238-44.

16. Langley RG, Ellis CN. Evaluating psoriasis with Psoriasis Area and Severity Index, Psoriasis Global Assessment and Lattice System Physicans Global Assessment. J Am Acad Dermatol 2004; 51: 563-9.

17. Reitan RM, Wolfson D. The Halstead-Reitan Neuropsychological Test Battery. Tucson, AZ: Neuropsychology Press 1985.

18. Stroop JR. Studies of interference in serial verbal reaction. J Exp Psychol 1935; 18: 643-62.

19. Beck AT, Ward CH, Mendelson M, et al. An inventory for measuring depression. Arch Gen Psychiatry 1961; 4: 561-71.

20. Julian LJ. Measures of anxiety: State-Trait Anxiety Inventory (STAI), Beck Anxiety Inventory (BAI) and Hospital Anxiety and Depression-Scale-Anxiety (HADS-A). Arthritis Care Res 2011; 63: 467-72.

21. Gurden H, Tassin JP, Jay TM. Integrity of the mesocortical dopaminergic system is necessary for complete expression of in vivo hippocampal-prefrontal cortex long-term potentiation. Neuroscience 1999; 94: 1019-27.

22. Bora E, Yücel M, Pantelis C. Cognitive impairment in affective psychoses: a meta-analysis. Schizophr Bull 2010; 36: $112-25$.

23. Schmitt J, Ford DE. Understanding the relationship between objective disease severity, psoriatic symptoms, illness-related stress, health-related quality of life and depressive symp- 
toms In patients with psoriasis - a structural equations modeling approach. Gen Hosp Psychiatry 2007; 29: 134-40.

24. Raison CL, Miller AH. The neuroimmunology of stress and depression. Semin Clin Neuropsychiatry 2001; 6: 277-94.

25. Schiepers OJ, Wichers MC, Maes M. Cytokines and major depression. Prog Neuropsychopharmacol Biol Psychiatry 2005; 29: 637-7.

26. Vollmer-Conna U, Fazou C, Cameron B, et al. Production of pro-inflammatory cytokines correlates with the symptoms of acute sickness behavior in humans. Psychol Med 2004; 34: 1289-97.

27. Joachim RA, Kuhlmei A, Dinh QT. Neuronal plasticity of the "brain-skin connection": stress-triggered up-regulation of neuropeptides in dorsal root ganglia and skin via nerve growth factor-dependent pathways. J Mol Med 2004; 85: 1369-78.

28. Whitley RJ, Meikle AW, Watts NB. Endocrinology. In: Tietz Textbook of Clinical Chemistry. $2^{\text {nd }}$ ed. Burtis CA, Ashwood ER (eds.). American Association for Clinical Chemistry Philadelphia. 1994; 1825-6.

29. Schmid-Ott G, Jaeger B, Meyer S, et al. Different expression of cytokine and membrane molecules by circulating lymphocytes on acute mental stress in patients with atopic dermatitis in comparison with healthy controls. J Allergy Clin Immunol 2001; 108: 455-62.

30. Evers AW, Verhoeven EW, Kraaimaat FW, et al. How stress gets under the skin: cortisol and stress reactivity in psoriasis. Br J Dermatol 2010; 163: 986-91.

31. Al Abadie MS, Kent GG, Gawkrodger DJ. The relationship between stress and the onset and exacerbation of psoriasis and other skin conditions. Br J Dermatol 1994; 130: 199-203.

32. Fordham B, Griffith C, Bundy C. Can stress reduction interventions improve psoriasis? A review. Psychol Health Med 2013; 18: 501-14.

33. Gisondi P, Sala F, Allessandrini F. Mild cognitive impairment in patients with moderate to severe chronic plaque psoriasis. Dermatology 2014; 228: 78-85. 